

\title{
Traces numériques et engagement du chercheur : contribution à une éthique de la communication en régime de controverse \\ Vincent Carlino, Grégoire Molinatti
}

\section{- To cite this version:}

Vincent Carlino, Grégoire Molinatti. Traces numériques et engagement du chercheur: contribution à une éthique de la communication en régime de controverse. Les Enjeux de l'information et de la communication, 2020, L'information scientifique, à l'épreuve de sa médiatisation, 2, pp.[En ligne]. 10.3917/enic.027.0013 . hal-02488398

\section{HAL Id: hal-02488398 \\ https://hal.univ-lorraine.fr/hal-02488398}

Submitted on 22 Feb 2020

HAL is a multi-disciplinary open access archive for the deposit and dissemination of scientific research documents, whether they are published or not. The documents may come from teaching and research institutions in France or abroad, or from public or private research centers.
L'archive ouverte pluridisciplinaire HAL, est destinée au dépôt et à la diffusion de documents scientifiques de niveau recherche, publiés ou non, émanant des établissements d'enseignement et de recherche français ou étrangers, des laboratoires publics ou privés. 


\section{Traces numériques et engagement du chercheur : contribution à une éthique de la communication en régime de controverse}

Article inédit, mis en ligne le 31 janvier 2020.

\section{Vincent Carlino}

Université de Lorraine, Centre de Recherche sur les Médiations et Université de la Réunion, Laboratoire de Recherche sur les Espaces Créoles et Francophones.

\section{Grégoire Molinatti}

Université de la Réunion, Laboratoire de Recherche sur les Espaces Créoles et Francophones et Centre Norbert Elias (AMU, EHESS, CNRS, Marseille).

\section{Plan de I'article}

Introduction

Une thèse qui engage le chercheur dans la controverse

Faire parler "une thèse qui fait parler"

L'enquête et la trace comme modalités d'engagement du chercheur

Références bibliographiques

Annexe : composition du corpus

\section{RÉSUMÉ}

Cet article porte sur la circulation sociale d'une thèse consacrée à l'histoire de l'administration de la preuve de sûreté d'un projet controversé de stockage de déchets radioactifs. L'analyse sémiotique des discours et des pratiques de médiation de la thèse permet d'en dégager des logiques, qui engagent le chercheur. Ce dernier ne peut donc faire l'économie d'une réflexion sur ses traces numériques et sur celles qu'il produit au sujet d'autres acteurs durant l'enquête. A partir des postures de chercheur adoptées dans nos propres travaux, notre recherche nourrit une éthique située de la communication des sciences en régime de controverse qui se focalise sur la légitimité des discours scientifiques et sur l'intentionnalité des traces numériques. Notre recherche contribue à développer des espaces de réflexivité au sein des sciences de l'information et de la communication (SIC).

\section{Mots clés}

Controverse, engagement, éthique, médiation scientifique, traces numériques.

\section{TITLE}

Digital traces and scientists' commitment: contribution to the ethics of communication in the context of public controversies 


\section{Abstract}

This paper is a case study analysis of the social discourses about a thesis focused on the story of the French nuclear waste management disposal reliability. It sheds light several logics of mediation of controversial scientific knowledge committing the scientist. We argue that the scientists can't avoid thinking about their digital traces and the traces they produce concerning other people during their fieldwork. At the light of our personal experiences, we contribute to the ethics of science communication. We focus on the legitimacy of scientific discourses in controversial contexts and on the intentionality of digital traces. In this perspective, ICS are invited to contribute to participate to the professional reflexivity.

\section{Keywords}

Controversy, commitment, ethics, science communication, digital traces

\section{Título}

Huellas digitales y implicación del investigador: contribución a una ética de la comunicación en contexto de controversia

\section{Resumen}

Este artículo trata de la circulación social de una tesis sobre la historia de la administración de la prueba de seguridad de un controvertido proyecto de almacenamiento de residuos radiactivos. El análisis semiótico de discursos y prácticas permite identificar lógicas de mediación científica que involucran al investigador. Por lo tanto, este último no puede evitar pensar en sus huellas digitales y en las que produce sobre otros actores durante la investigación. Desde la postura de investigador adoptada en nuestro propio trabajo, nuestra investigación alimenta una ética de la comunicación de la ciencia en contexto de controversia. Nuestro artículo se focaliza en la legitimidad de los discursos científicos y en la intencionalidad de las huellas digitales. Contribuye a desarrollar espacios de reflexividad dentro de las ciencias de la información y la comunicación.

\section{Palabras clave}

Controversia, ética, huellas digitales, implicación, mediación científica

\section{INTRODUCTION}

"J'envisageais d'écrire un livre, de rendre public le manuscrit. Maintenant qu'il l'est, je ne vois plus trop l'intérêt. " C'est ainsi qu'un chercheur en Sciences Humaines et Sociales (SHS) venant de soutenir une thèse témoigne de son sentiment de ne pas maîtriser la médiatisation de son travail scientifique. Dans quelle mesure la médiatisation engage-t-elle ce chercheur prenant pour objet de recherche une controverse sociotechnique? Le cas de la circulation sociale d'une thèse d'histoire des sciences consacrée à la gestion des déchets radioactifs permet de rendre compte des logiques de médiation dans lesquelles le chercheur se trouve engagé. Prenant acte des jeux médiatiques inédits que soulève la mise en média de l'information scientifique, cet article entend contribuer à la réflexion sur les enjeux de l'exposition systématique des chercheurs sur les plateformes en ligne. En se focalisant sur les logiques de médiatisation en régime de controverse, il entend discuter la place des chercheurs dans le débat public à la lumière de l'intentionnalité de leur communication. L'engagement des chercheurs, que nous avons par ailleurs analysé à partir d'autres études de cas de communication d'expertises - utilisation des cellules souches embryonnaires humaines, exploitation des gaz de schistes, ouverture de la procréation médicalement assistée aux couples de même sexe (Birgé \& Molinatti, 2019), — est ici saisi à l'aune des 
traces numériques liées aux pratiques de recherche. Dans cette perspective, nous souhaitons contribuer à une éthique de la communication à partir de nos propres expériences d'enquête sur des controverses d'environnement et de santé.

La gestion politique des déchets radioactifs dont il est question dans la thèse mobilise, depuis son émergence, une expertise scientifique et technique. Avec le «tournant participatif » (Lehtonen, 2011) des années 1990, les sciences humaines et sociales sont considérées comme légitimes pour aider à traiter les « aspects sociaux » du nucléaire. L'intervention des chercheurs en sciences humaines et sociales vise à "réduire le problème social $d u$ nucléaire à une question de communication ou de collaboration entre différents groupes sociaux » (Lits, 2014, p.111). La dimension communicationnelle de l'expertise apparaît centrale dans cette controverse qui se cristallise aujourd'hui autour du projet du Centre industriel de stockage géologique (Cigéo) piloté par l'Agence nationale pour la gestion des déchets radioactifs (Andra). C'est dans ce contexte qu'est soutenue le 11 décembre 2017 la thèse de doctorat intitulée "Enfouir des déchets nucléaires dans un monde conflictuel. Une histoire de la démonstration de sûreté de projets de stockage géologique, en France (1982-2013) " (Patinaux, 2017).

Dès sa soutenance publique, la thèse circule entre des scientifiques et des membres de l'Andra, mais aussi des opposants au projet Cigéo et des journalistes, donnant lieu à un ensemble de médiations et de discours «à propos de sciences»(Cheveigné, 1997). Afin de rendre compte des processus de sémiotisation à l'œuvre, nous envisageons ces discours en rupture avec un modèle linéaire de transfert de savoirs, du savant au profane, et les logiques de traduction ou de trahison que cette perspective suppose. Nous nous focalisons sur la dimension argumentative de ces discours et sur les stratégies d'acteurs qui les produisent et les mobilisent (Chateauraynaud, 2011). À cette fin, les principaux auteurs des discours retenus dans un corpus (cf. annexe) ont été interrogés sur les enjeux et les modalités de leurs pratiques discursives, leurs relations aux autres acteurs impliqués, et plus généralement sur la place de l'expertise scientifique dans les controverses publiques ${ }^{1}$. L'attention portée à la matérialité des discours circulants (Le Marec \& Babou, 2015) et aux pratiques et représentations des acteurs, situe notre approche en anthropologie de la communication. L'expertise scientifique est ici envisagée comme une activité de médiation entre sciences et décision (Roqueplo, 1997) mettant en jeu des assemblages " de processus, de pratiques et d'objets conduisant à des situations particulières de pouvoir » (Quet, 2014). Penser une «situation d'expertise» (Bouillon, 2012) scientifique dans une perspective communicationnelle suppose donc d'interroger les relations entre les parties prenantes et les cadres institutionnels qui travaillent sa légitimité et les dimensions symboliques, médiatiques et sociotechniques qui la rendent visible et la transforment.

\section{UNE THÈSE QUI ENGAGE LE CHERCHEUR DANS LA CONTROVERSE}

La thèse dont nous interrogeons la circulation sociale se présente comme relevant d'une histoire (sociale) des sciences. Ses partis pris épistémologiques envisagent la dimension cognitive des savoirs scientifiques comme "l'avers non séparable d'un revers constitué de pratiques sociales qui contribue à définir le bon savoir, la bonne preuve »(Pestre, 2001, p.185). Ainsi, elle

1. L'auteur de la thèse, un opposant ayant participé à sa médiation ainsi que les journalistes nous ont accordé en toute confiance des entretiens d'une durée d'1h à $1 \mathrm{~h} 30$. Le directeur de la recherche et du développement de l'Andra nous a accordé un entretien téléphonique en compagnie de la responsable de la communication, à la condition préalable que nous leur fassions parvenir les questions avant l'entretien (ce que nous avons fait) et qu'ils puissent relire notre production avant sa publication (ce que nous avons refusé pour d'évidentes raisons déontologiques, nous engageant seulement à tenir à leur disposition l'enregistrement de nos échanges). 
propose une mise en perspective historique de la façon dont l'Andra travaille la preuve de sûreté de son projet depuis les années 1980. Écrite par un historien des sciences, la thèse avance que pour la période actuelle :

"Il n'y a pas de preuve ni de démonstration de la sûreté d'un stockage mais l'Andra a développé, en relation avec d'autres institutions, tout un ensemble de pratiques qui l'informent sur l'évolution de l'ouvrage et doivent lui permettre de convaincre (et de se convaincre) qu'elle maîtrise le projet qu'elle conçoit. Lorsque l'Agence doit produire une analyse de sûreté globale, l'arrangement des savoirs produits en un ensemble cohérent montre néanmoins un certain bricolage » (Patinaux, 2017, p.410, Conclusion générale).

L'idée directrice de la thèse est ainsi posée : il s'agit de montrer comment l'Andra construit et met à l'épreuve une argumentation visant à convaincre de la sûreté du projet Cigéo. L'accès aux archives de l'Andra, comme la possibilité donnée au chercheur d'assister à certaines réunions de travail internes lui ont permis d'observer les pratiques sociales de construction de l'expertise sur laquelle repose cette argumentation. Nous retenons trois dimensions qui questionnent l'engagement du chercheur dans son travail empirique et analytique.

D'abord, il est mentionné dans la thèse que "l'Andra a indiscutablement accumulé une grande quantité de savoirs sur l'évolution d'un projet de stockage et [que] de nombreuses connaissances sont stabilisées " (Ibid., p. 356). Mais la démonstration de sûreté est relativisée et se concentre sur la gestion des incertitudes qui subsistent bien qu'elles soient rendues « visibles ». C'est par exemple le cas de l'" évaluation de la vitesse de dégradation du verre » dans lequel sont piégés les radionucléides. L'auteur rapporte qu' "il est soulevé [par les employés de l'Andra, $\mathrm{ndr}$ ] que la démonstration de sûreté ne sera pas une démonstration mathématique mais davantage la production d'un "faisceau d'arguments " qui doit permettre de justifier que le stockage se comportera bien comme prévu» (Ibid., p.370).

Ensuite, la thèse étant financée par l'Andra, elle expose son auteur à des accusations de potentiels conflits d'intérêts. L'auteur de la thèse revendique une approche critique soutenant que tous les aspects du nucléaire sont sociaux. À l'inverse, l'Andra, se situe dans un paradigme de décision neutre et fondée sur les sciences (Andra, 2010, p.8).

Enfin, la thèse rend compte (dès l'introduction, puis dans la partie 3 du chapitre 6 intitulée "Sciences sociales et gouvernance de Cigéo ») de la mobilisation des sciences sociales dans l'expertise autour de l'enfouissement des déchets radioactifs. Elle rappelle de quelle manière des chercheurs ont introduit puis accompagné le « tournant participatif » à partir des années 1990. L'auteur analyse en particulier le travail des sociologues du Comité d'Expertise et de Suivi de la Démarche d'Information et de Consultation (Coesdic), chargé de conseiller la direction de l'Andra. Il signale la manière dont ils opèrent une " traduction " en plébiscitant le terme d' "entreposage duquel il serait possible de ressortir les déchets » et non de «stockage». Selon l'auteur, cette traduction doit "être comprise comme une tentative de désinhibition du recours à l'évacuation géologique des déchets nucléaires » (p.331) chère aux opposants. Pour autant, il ne questionne pas les choix sémantiques qu'il a lui-même opérés (il utilise le terme de stockage), bien qu'ils aient fait l'objet d'une négociation avec les relecteurs de l'Andra. De plus, l'auteur n'interroge pas la façon dont sa thèse pourrait être prise elle-même dans un «faisceau d'arguments » qui viserait à prôner la faisabilité de Cigéo. Autrement dit, la relativisation de la démonstration de sûreté ainsi que le financement de la thèse par l'Andra engagent son auteur. Cependant, cet engagement n'est pas discuté dans le manuscrit alors même que plusieurs passages critiquent la mobilisation des sciences sociales dans la gestion des déchets radioactifs (p.323-344). Ce sont d'autres acteurs de la controverse qui vont se charger de définir l'engagement du chercheur. 


\section{FAIRE PARLER « UNE THÈSE QUI FAIT PARLER »}

Peu après sa soutenance, la thèse fait l'objet de commentaires médiatisés dont il convient de tracer la généalogie. Soutenue le 11 décembre 2017, la thèse est repérée par un opposant à Cigéo qui publie un communiqué de presse en ligne intitulé "Cigéo Papers ${ }^{2}$ le 5 février 2018. En la partageant, les acteurs font parler la thèse : ils en dégagent les idées principales et livrent des clés de lecture et d'interprétation. Ainsi la parole du chercheur se confronte-t-elle aux pratiques communicationnelles de ceux qui s'en emparent. C'est ce qui se produit avec ce communiqué qui divulgue des «fuites» et lance l'alerte avec des formules telles que "nous venons d'apprendre de source sûre » ou encore « le document que nous nous sommes procuré est authentique» (italiques de l'auteur). Le lendemain, Le Monde consacre un article à la thèse qui, dès le titre, estime qu'elle démontre "l'impossible preuve scientifique de la sûreté » de Cigéo. Il est suivi par Le Journal de la HauteMarne qui titre "Cigéo : une thèse qui fait parler » et met l'accent sur le rôle d'opposants qui «rend[ent] publics des propos exprimés en interne par des salariés de l'Andra ». À l'échelle locale, la médiatisation met l'accent sur l'alerte des opposants. En revanche, les médias nationaux traitent la controverse dans sa globalité, soulignant ce que cette thèse apporte au débat. C'est dans le cadre d'une émission de France Culture en février 2018 que l'auteur de la thèse s'exprime pour la première fois. Il réitère l'exercice quelques mois plus tard dans une interview pour le média Grozeille.co. Enfin, son audition le 31 mai 2018 devant la Commission d'enquête sur la sûreté et la sécurité des installations nucléaires marque l'entrée de la thèse dans un régime d'expertise à finalité politique. Cette thèse a donc été largement médiatisée. Pourtant, une partie des personnes interrogées lors de nos entretiens expriment l'espoir déçu que celle-ci n'ait pas produit un effet politique plus important. Par l'extraction d'extraits choisis, ces acteurs ont essayé de faire parler la thèse en l'intégrant dans leurs logiques. Il convient donc de préciser ces logiques et les pratiques communicationnelles qui les servent.

\section{Usages des citations dans les logiques d'acteurs}

Commençons par l'auteur de la thèse qui explique ne pas avoir intégré en amont la question de la publicisation de son travail. L'idée d'une déformation des propos par l'extraction de citations employées comme des formules (Krieg-Planque, 2009) revient dans les échanges avec l'auteur, renvoyant à ce qu'il perçoit comme une forme de trahison du propos scientifique (Jurdant, 2009). On peut citer l'exemple de l'extrait médiatisé : "la démonstration de sûreté de Cigéo ne s'apprécie pas en fonction de sa justesse, mais en fonction de sa capacité à convaincre ses évaluateurs» (Le Monde, France Culture, Revue Générale Nucléaire). Face à ce qu'il juge être des réductions de son propos, le chercheur adopte une posture réactive où il explicite sa démarche de recherche. Il insiste sur son approche historique de la gestion des déchets radioactifs depuis 1980, de même qu'il rappelle que le scénario " est un outil classique des ingénieurs pour appréhender le futur »3. Cette stratégie est perçue par les autres acteurs comme une euphémisation de ce qu'ils pensent être le propos central de la thèse, une volonté de son auteur de calmer la controverse.

L'Andra est aussi concernée à la fois en tant qu'objet et financeur de la thèse. Cette recherche est conçue comme une contribution à l'amélioration de l'administration de la

2. Dans les jours et les mois qui suivent, les «Cigéo Papers » sont relayés par nombre de sites écologistes et antinucléaires (dont Anti-K, Savoie Antinucléaire, Cade-environnement, Résistance verte, Aura-Environnement, Observatoire du nucléaire, Attac-54, Burestop) et commentés sur des sites de presse d'information (dont Le Club Mediapart, Républicain Lorrain, L'Est Républicain, Le Journal de la Haute Marne, Libération).

3. Source : https://grozeille.co/bure-economie-risque-nucleaire/ consulté le 21 février 2019. 
preuve de sûreté qui permet aux employés de questionner la prise en compte de leurs incertitudes. De plus, les lecteurs de la thèse ne se limitent pas à la communauté des pairs. Elle sert à expliquer aux non spécialistes le travail d'expertise des ingénieurs de l'Andra en vue de la demande d'autorisation de construction de Cigéo. Le premier discours public apparaît dans un article de la Revue Générale Nucléaire qui donne la parole à Frédéric Plas, directeur de la recherche et du développement de l'Andra. Lors d'un entretien en janvier 2019, ce dernier explique que l'Andra a financé la thèse "dans un objectif de transparence, de rigueur scientifique et d'amélioration de ses bases de processus de décision ».

L'Andra ne s'exprime sur le contenu de la thèse qu'à la suite de la publication des « Cigéo Papers » par un opposant affirmant avoir obtenu des informations exclusives et compromettantes. En réalité, dans les « Cigéo Papers ", l'interprétation des propos de l'auteur de la thèse et le sens qui leur est donné s'opèrent en deux temps. D'abord, l'opposant est à l'origine de la médiatisation de la thèse par une mise en ligne « sauvage » sur son site web. Il perçoit son auteur comme un lanceur d'alerte qui, du fait de son immersion de trois ans à l'Andra, a eu accès à des documents sensibles et confidentiels. L'opposant se pose en relais de ce qu'il interprète comme une alerte qui lui sert à se "confronter » à l'Andra « en tant qu'instance normativeCigéo ». L'exploitation du manuscrit a ainsi donné lieu aux « Cigéo Papers ", à savoir une sélection de citations, mais aussi à une analyse sollicitée auprès d'un scientifique opposé au projet. Cette pratique s'inscrit dans « l'utopie délibérative » de la mouvance antinucléaire qui vise à militer "pour réintroduire des pratiques plus démocratiques dans le processus politique de développement de l'énergie électronucléaire " (Chambru, 2015, p.61). Comme nous le discuterons par la suite, cette première étape interroge le chercheur sur le fait qu'il perd la maîtrise de ses arguments ainsi que celle de la diffusion du texte intégral de son manuscrit. Les acteurs qui prennent en charge la publicisation de la thèse engagent donc le chercheur malgré lui. Dans un second temps, l'initiateur des " Cigéo Papers " porte un regard réflexif sur sa propre communication et revoit son jugement. Il ne considère plus la thèse " comme une bombe ", mais comme une opération "montée de toute pièce pour faire dire [que l'Andra] ne peut pas démontrer mathématiquement la faisabilité de Cigéo » comme il l'affirme dans un entretien avec les auteurs de cet article en janvier 2019. Cette nouvelle lecture met en cause la posture du chercheur. Celui-ci est dépeint comme un artefact servant à porter et à légitimer scientifiquement un message que l'Andra ne pourrait pas diffuser dans sa communication institutionnelle. Jugeant s'être « fait avoir », l'opposant requalifie l'alerte en «coup de com' remarquable »:

"Ce que nous avons appelé les CIGéo-Papers font partie en fait de la construction de l'acceptation du projet CIGéo. Au lieu d'avouer qu'elle est incapable de démontrer mathémathiquement la faisabilité de CIGéo et encore moins la maîtrise des risques, l'ANDRA l'a fait dire par un thèsard! " ${ }^{4}$.

Après s'être appuyé sur une partie du contenu de la thèse, l'auteur des «Cigéo Papers » passe de l'alerte à l'instrumentalisation des SHS visant à favoriser l'acceptabilité de son projet. Ce revirement énonciatif est pourtant absent de la médiatisation de la thèse, et notamment de l'article du Monde qui la considère comme "un document embarrassant pour les promoteurs » de Cigéo. Cet article est important car il fait lui aussi circuler des extraits choisis. Le propos se concentre sur «l'impossible preuve scientifique de la sûreté » de Cigéo qui abandonne le modèle mathématique au profit d' "un faisceau d'arguments » sur lequel repose un «scénario » conçu «comme on raconte une histoire ». Les citations portent surtout sur le «bricolage» (au sens péjoratif du terme) et sur le «toilettage » des documents internes qui convergent vers l'idée d'un arrangement des savoirs. La question du public

4. Guéritte, Michel (2018), CIGéo Papers. 5 février, [en ligne], https://www.villesurterre.eu/index.php?option=com_content\&view=article\&id=634:cigeo-papers\&catid=124:news\&Itemid=214 
est centrale ici, car il s'agit de montrer aux lecteurs comment se construisent et s'administrent les preuves de sûreté. La seule lecture de l'article laisse supposer que son auteur considère ces informations comme des révélations. Cependant, l'entretien avec l'auteur, journaliste à la rubrique « Planète » du Monde, révèle une posture plus fine à l'égard de son public, qui est pensé " dès les étapes liminaires de production des contenus » (Ballarini \& Ségur, 2018, p.11). En effet, le journaliste a fait « expertiser» la thèse par un membre de l'Institut de radioprotection et de sûreté nucléaire (IRSN) avant de la commenter. Pour ce journaliste, la scientificité du document repose sur une expertise qu'il prend l'initiative d'organiser. Cette posture critique à l'égard des sciences le pose comme médiateur dans le débat entre experts. Cette expertise fait écho aux pratiques professionnelles du journaliste en matière de vérification et de protection des sources pour livrer à son public une information qu'il juge crédible et légitime.

Cette considération du public se retrouve aussi chez la journaliste de France Culture qui a invité l'auteur de la thèse à son émission grand public Dimanche et après? du 11 février 2018. La journaliste souhaite créer un débat ouvert et contradictoire sur le sujet de l'enfouissement des déchets nucléaires. Lors d'un entretien que nous avons mené en janvier 2019 avec cette journaliste, celle-ci suppose que l'auteur a accepté de s'exprimer à l'antenne pour "nuancer ce qui s'est dit dans l'article du Monde ». L'émission radiophonique est ici pensée comme un espace de parole permettant au chercheur de lever les ambigüités nées de la médiatisation de sa recherche. L’organisation du débat contradictoire pose la journaliste comme médiatrice entre les différents intervenants dans l'intérêt de son public. Elle formule ses attentes quant à l'émergence d'un tel débat :

"En tant que journaliste, je ne vois pas pourquoi je ne croirais pas un intervenant qui me dit qu'il a voulu faire un travail d'histoire et que Le Monde l'a utilisé plus ou moins à des fins de contestation. Ça m’intéresse qu'il vienne dire ça. Je trouve que ça pose question, après je fais confiance aux auditeurs pour eux-mêmes savoir ce que ça signifie."

L'échange porte non seulement sur le contenu de la thèse, mais aussi sur les intentions de ceux qui la médiatisent - y compris du chercheur qui se livre à l'exercice en plateau. La journaliste considère que le chercheur ne va pas assez loin dans sa réaction à l'article du Monde qu'elle présente comme "fais[ant] un peu froid dans le dos ». Toutefois, elle s'estime satisfaite de l'émission car l'invité a fait preuve de nuance : "ça changeait, c'était quelqu'un qui venait exposer sa thèse. »

\section{Le chercheur à l'épreuve des (non) pratiques de communication scienti- fique}

Si elles apparaissent hétérogènes, les logiques des médiations de la thèse traduisent des stratégies argumentatives adaptatives qui interrogent quant à une éventuelle stratégie de communication scientifique du chercheur. Comment se positionne-t-il dans les logiques d'acteurs, alors qu'il est attendu par certains pour peser sur le débat et, par d'autres, pour le donner à voir ou l'organiser? Quelles que soient leurs logiques, les pratiques de communication des acteurs demeurent bricolées, si bien qu'ils partagent le sentiment de ne pas les maîtriser. C'est le cas de l'opposant qui, pensant découvrir un lanceur d'alerte, révise son jugement. L'absence de maîtrise se lit aussi chez le journaliste du Monde qui s'empresse de publier l'affaire tout en sachant que Le Canard Enchaîné a aussi connaissance de la thèse, ainsi que la journaliste de France Culture qui espérait que l'auteur de la thèse aurait contredit ou confirmé les différentes (re)formulations médiatiques de sa recherche. Le chercheur peut paraître isolé face aux acteurs qui publicisent, interprètent et reformulent les résultats de son travail. 
La mise en circulation du contenu de la thèse semble indissociable de la construction médiatique de l'identité de son auteur. Les « Cigéo Papers » en offrent un bon exemple. En effet, l'opposant présente l'auteur de la thèse comme un avatar communicationnel servant à porter un message institutionnel. L'accusation porte sur le chercheur, présenté comme suspect car n'ayant "pas une seule photo de lui sur internet ". La suspicion va plus loin, car l'absence de traces numériques amène l'opposant à imaginer que le chercheur ait pu être «construit » par l'Andra. Il s'engage alors dans la collecte et la publicisation d'informations pour façonner l'identité numérique du chercheur à son insu. C'est ainsi qu'une photographie de l'auteur se trouve publiée sur les "Cigéo Papers ", de même que les profils des membres du jury de thèse.

La médiation des sciences donne une autre portée aux données de recherche. En effet, la production scientifique repose sur des normes de mise à disposition des données empiriques. En l'occurrence, le chercheur rend visible des documents d'archives de l'Andra qui composent son corpus. Lorsque la thèse circule entre plusieurs arènes (Badouard, Mabi, Monnoyer-Smith, 2016), elle attire l'attention d'autres acteurs sur certains documents sensibles qu'ils n'auraient pas repérés autrement. C'est le cas d'un opposant qui assigne l'Andra à lui envoyer une partie de ses archives repérées dans le manuscrit. Pour en revenir à l'auteur de la thèse, le chercheur, en indiquant ses sources, nourrit indirectement la critique.

Ainsi, le chercheur s'engage dans les médiations opérées autour des usages sociaux de ses activités de recherche et se trouve également enrôlé dans ces médiations. Cependant, l'hétérogénéité des logiques et des pratiques de communication ruine l'illusion d'une quelconque possibilité de maîtrise de la communication publique de leurs productions. Même l'absence de communication est susceptible d'être interprétée comme une stratégie. Ce qui n'empêche pas le chercheur d'avoir à penser ses formes d'engagement par la communication.

\section{L'ENQUÊTE ET LA TRACE COMME MODALITÉS D'ENGAGEMENT DU CHERCHEUR}

L'approche communicationnelle confirme qu'il paraît impossible de travailler une controverse sociotechnique sans s'y engager. La séparation entre la publication scientifique d'une part et ses médiations symboliques et sociotechniques d'autre part semble intenable. En fait, l'engagement du chercheur se joue dès le temps de l'enquête qui implique des rapports sociaux avec les acteurs qui prennent en compte la dimension institutionnelle de la recherche.

La question de l'engagement du chercheur en sciences humaines et sociales a déjà été largement explorée. Elle a été posée par Max Weber (1963) qui défend l'incompatibilité entre les domaines politique et scientifique. Cette question a donné lieu à des "propositions normatives » (Bourdieu, 1997) dont celle de Nathalie Heinich en faveur d'une " neutralité engagée » (2002) qui consiste à expliciter les logiques des acteurs pour éclairer le débat. À l'inverse, Irène Théry pense son « expertise d'engagement » (2005) comme des " propositions pour l'action» sur lesquelles il s'agit "d'argumenter clairement une option possible parmi d'autres ». La sociologie des controverses ne reste pas en marge. Les chercheurs ont interrogé les usages politiques de leurs expertises, proposant une « co-production » des savoirs et des normativités sociales (Jasanoff, 1996).

Pour notre part, nous envisageons l'engagement non pas sous l'angle de la neutralité, mais sous celui des transformations des objets d'étude des scientifiques par la recherche. Ce choix s'inspire de l'exemple des sociologues de l'innovation qui ont transformé leur 
objet en "tradui[sant] les questions de choix nucléaires techniques en question d'organisation des formes de société » (Lits, 2014, p.122).

Qu'en est-il du point de vue des sciences de l'information et de la communication? On pourrait par exemple se demander dans quelle mesure les analyses des dispositifs de médiation des sciences engagent leurs auteurs, qui soulignent la tension entre valorisation et mise à distance critique. On pourrait aussi interroger l'engagement des chercheurs qui œuvrent à " prendre l'engagement citoyen au sérieux 》 (Chavot \& Masseran, 2010, §39) et mettent à distance le modèle du déficit de connaissances des publics dans les dispositifs de médiation scientifique "à mi-chemin du Public Engagement in Science " (Ibid.). Cependant, il paraît difficile d'évaluer la portée de ces travaux sur l'évolution des pratiques de médiation des sciences. C'est pourquoi nous abordons l'engagement des chercheurs en sciences de l'information et de la communication à partir des notions d'enquête et de trace. Comme le rappelle Joëlle Le Marec (2002), « la communication est tout à la fois l'objet, la méthode, et l'extérieur de la méthode, le monde de sens commun contre lequel elle se pose ». Les chercheurs en SIC ont pour spécificité d'enquêter sur des traces de communication et de laisser à leur tour des traces sur leurs terrains. À la lumière de nos enquêtes et de nos expériences de communication de recherche dans l'espace public, cette dialectique de circularité entre l'enquête et la trace nous amène à préciser trois éléments pour travailler une éthique de la communication de la recherche en régime de controverse à partir de cas concrets.

En premier lieu, la publicisation de la recherche gagne à être resituée parmi une pluralité d'expertises. De fait, lorsque le chercheur médiatise son travail, des acteurs de la controverse essaient de le situer parmi l'ensemble des discours et des connaissances à leur disposition. C'est ce que l'un d'entre nous a éprouvé à la suite de la publication d'un article sur la contestation à Bure pour le site d'information The Conversation France ${ }^{5}$. Des lecteurs ont investi les commentaires pour affirmer que l'article était à charge puisqu'il faisait référence à l' "enfouissement » et non au "stockage » des déchets radioactifs. En parallèle, l'article était relayé sur des sites antinucléaires. Faute d'avoir été clairement exposé, le positionnement du chercheur était alors défini par les autres acteurs. Cela invite le chercheur à relativiser la légitimité sociale des savoirs scientifiques tout en communicant sur leur spécificité. Nous pourrions imaginer que le chercheur explique la place qu'occupe son travail dans la discipline (à partir des expertises qu'il a connues : comités de lecture, jurys, etc.) et plus largement dans une pluralité de discours sociaux. La communication des chercheurs ne se limite alors pas à expliquer sa posture épistémologique (comme nous l'avons vu précédemment dans l'étude de cas), mais à situer sa parole et sa légitimité par rapport à d'autres.

Par ailleurs, le chercheur n'est pas seul enquêteur. Ainsi, nous avons constaté que nos informateurs accordent (ou non) des entretiens en ayant le plus souvent enquêté sur nos propres traces numériques de chercheurs en SIC. C'est notamment le cas des «citoyens-enquêteurs » (Carlino, 2019) et des institutions parties prenantes de la controverse. L'identité du chercheur, son affiliation institutionnelle, l'origine de ses financements, ses collaborations, ses engagements politiques, ses apparitions médiatiques, etc. sont autant d'éléments que les personnes interrogées interprètent et qui conditionnent l'enquête ainsi que la circulation sociale des résultats qui en sont tirés. Souvent produites par des tiers, au sein de dispositifs qui peuvent dépasser le cadre professionnel, les traces numériques

5. Carlino, V. (2017) Déchets nucléaires : comprendre l'escalade de la violence autour du projet Cigéo. Accès : http://theconversation.com/dechets-nucleaires-comprendre-lescalade-de-la-violence-autour-du-projetcigeo-83114 
apparaissent très tôt dans le parcours de recherche (par exemple lors de l'inscription du sujet de thèse dans la base de données nationale). La communication du chercheur ne désigne plus seulement la production volontaire de contenu, mais s'oriente vers la gestion de son identité numérique (Ertzscheid, 2013 ; Gomez-Mejia, 2016). Du point de vue de l'engagement, cela nécessite de méta-communiquer pour « intentionnaliser » les traces (Merzeau, 2013, p.122). Ainsi, la communication des chercheurs ne porte plus seulement sur le contenu de leurs travaux à des fins de vulgarisation et de diffusion. Ces derniers doivent s'exprimer publiquement en vue de contester ou de valider les commentaires médiatiques et les usages sociaux de leurs activités de recherche. C'est par cette communication sur les médiatisations de leurs travaux que les chercheurs s'engagent dans les controverses.

Par l'enquête de terrain et sa restitution, les chercheurs produisent aussi des traces sur leurs enquêtés. Par exemple, notre enquête a amené les personnes interrogées à réécouter, à relire et donc à penser les discours qu'ils avaient produits un an plus tôt. Avec les divers dispositifs numériques, l'éthique de l'enquête (publication, confidentialité des données, anonymisation, consentement éclairé) est en partie à réinterroger (Simonnot, 2018). Pour penser leur engagement par la communication, les chercheurs doivent donc prendre conscience des traces à leur sujet, ainsi que de celles qu'ils produisent sur d'autres acteurs. Il reste à mener un travail de recherche empirique sur la façon dont les chercheurs pensent et travaillent leurs traces numériques.

Enfin, l'impossibilité de maîtriser sa communication pourrait justifier la tentation de déléguer la publicisation de ses travaux à des professionnels. D'autant que les principales institutions scientifiques se sont dotées de services de communication. Souvent inscrits dans des logiques de marchandisation de la science, ces services se donnent pour mission de maîtriser la communication scientifique et les « risques et opportunités » qui pourraient être liés à une mise en visibilité des sciences. Au-delà des logiques d'autonomisation de la communication scientifique (Babou \& Le Marec, 2008), la délégation de la communication à des professionnels s'apparente selon nous à une forme de désengagement. Ce qui pourrait donner aux chercheurs l'illusion de faire l'économie d'une réflexion sur leur engagement.

Une telle posture ne semble pas tenable. En effet, les dynamiques de controverse et d'enquête poussent les chercheurs à envisager la portée sociale de leur communication (Molinatti \& Simonneau, 2015) comme une forme de responsabilité sociale qui engage aussi celle de leurs institutions (Appel \& Falgas, 2018). Les espaces de discussion et de réflexion collective sur ces questions restent rares. Et les formations doctorales consacrées à la communication répondent souvent à une injonction adressée aux jeunes chercheurs dans une perspective de valorisation de leurs travaux, à travers la maîtrise de dispositifs standardisés (à l'instar du concours $M a$ thèse en 180 secondes). Il semble donc nécessaire d'engager un travail collectif sur nos pratiques communicationnelles. Il ne serait pas question de les maitriser mais de comprendre de quelle manière, en contexte, elles se déploient, et d'en envisager la portée. Cette visée assume une forme de «bricolage » entendu comme un «savoir-faire » parfois assumé sur le plan épistémologique (Olivier de Sardan, 2008, p.44).

Depuis plusieurs décennies, les sciences de l'information et de la communication ont consacré des travaux aux médiations des sciences. Elles en ont souligné les enjeux, analysé les discours, observé les pratiques et les représentations, mis au jour les logiques et stratégies d'acteurs. À ces aspects, doit s'ajouter l'engagement des chercheurs par les médiatisations de leurs travaux. Sans réduire l'engagement aux seules problématiques de communication, il nous semble important que les SIC contribuent aux espaces de formation et de réflexivité des chercheurs, particulièrement dans un contexte où les politiques 
d'évaluation de la recherche poussent les chercheurs (y compris les doctorants, comme en témoigne l'opération Ma thèse en 180 secondes par exemple) à prendre eux-mêmes en charge la médiation et la médiatisation de leurs travaux. C'est aussi une façon de nourrir une « critique des sciences » (Quet, 2008) ; les approches communicationnelles ayant, dès leur origine, participé de ce mouvement.

\section{RÉFÉRENCES BIBLIOGRAPHIQUES}

Andra (2010), Rendre gouvernables les déchets radioactifs. Le stockage profond à l'épreuve de la réversibilité. Rapport n ${ }^{\circ} 381$, Châtenay-Malabry : Andra, [en ligne], Consulté le 18 octobre 2019, https://www.andra.fr/sites/default/files/2017-12/381.pdf.

Appel, Violaine ; Falgas, Julien (2018), « Responsabilité sociétale des universités et environnement numérique. La notion d'empreinte, un enjeu de réflexivité », Communication, $\mathrm{n}^{\circ} 35(2)$.

Babou, Igor ; Le Marec, Joëlle (2008), « Les pratiques de communication professionnelle dans les institutions scientifiques ", Revue d'anthropologie des connaissances, ${ }^{\circ} 2$ (1), p.115142.

Ballarini, Loïc ; Ségur, Céline (coord.) (2018), Devenir public. Modalités et enjeux, Paris : mare \& martin.

Birgé, Robin ; Molinatti, Grégoire (2019), "Neutralisation et engagement dans des controverses publiques. Approche comparative d'expertises scientifiques » (p.291-309), in Florence Piron ; Mélissa, Lieutenant-Gosselin ; Laurence Brière (dir.) Et si la recherche scientifique ne pouvait pas être neutre? Québec : Editions Sciences et bien commun.

Bouillon, Jean-Luc (2012), «L'expertise scientifique en société : regards communicationnels », Hermès, n 64 , p. 14-21.

Bourdieu, Pierre (1997), Les usages sociaux de la science. Pour une sociologie clinique du champ scientifique, Versailles : Éditions Quæ.

Carlino, Vincent (2019), L'énergie de la contestation : formes de désaccord et arènes du conflit sur le nucléaire en Lorraine. Thèse en sciences de l'information et de la communication, Metz : Université de Lorraine, [en ligne], Consulté le 18 octobre 2019, https://hal.univ-lorraine. $\mathrm{fr} / \mathrm{tel}-02090208$.

Chambru, Mikaël (2015), "L’utopie délibérative de la mouvance antinucléaire et les paradoxes de son expérimentation », Communication Eं Organisation, n 48, p. 61-72.

Chateauraynaud, Francis (2011), Argumenter dans un champ de forces. Essai de balistique sociologique, Paris : Pétra.

Chavot, Philippe ; Masseran, Anne (2010), «Engagement et citoyenneté scientifique : quels enjeux avec quels dispositifs ? ", Questions de communication, ${ }^{\circ}$ 17, p. 81-106.

Cheveigné, Suzanne (1997), "La science dans une société médiatisée », Hermès, n²1, p. 15-22.

Ertzscheid, Olivier (2013), Qu'est-ce que l'identité numérique ?: Enjeux, outils, méthodologies. Encyclopédie numérique. Marseille : OpenEdition Press.

Gomez-Mejia, Gustavo (2016), Les fabriques de soi ? Identité et industrie sur le web, Paris : MkF éditions.

Heinich, Nathalie (2002), "Pour une neutralité engagée », Questions de communication, $\mathrm{n}^{\circ} 2$, p. $117-127$. 
Jasanoff, Sheila (1996), "Beyond Epistemology: Relativism and Engagement in the Politics of Science », Social Studies of Science, n 26 (2), p. 393-418.

Jurdant, Baudouin (2009), Les problèmes théoriques de la vulgarisation scientifique. Paris : Archives contemporaines.

Krieg-Planque, Alice (2009), La notion de formule en analyse du discours : cadre théorique et méthodologique, Besançon : Presses universitaires de Franche-Comté.

Le Marec, Joëlle (2002), «Situations de communication dans la pratique de recherche : du terrain aux composites ", Études de communication, n ${ }^{\circ} 25$, p. 15-40.

Le Marec, Joëlle ; Babou, Igor (2015), « La dimension communicationnelle des controverses ", Hermès, n 73, p. 111-121.

Lehtonen, Markku (2011), «Le «tournant participatif» dans la gouvernance nucléaire en Finlande, en France et au Royaume-Uni : des antécédents historiques aux enjeux actuels ", in Actes de la Journée d'études sur les effets de la participation, Paris : Gis Démocratie et participation [en ligne], http://www.participation-et-democratie.fr/sites/default/files/ atelier 3-1 lehtonen.pdf, consulté le 12 mars 2019.

Lits, Grégoire (2014), « Curiosité ou engagement? Panorama historique du rôle des chercheurs en sciences sociales dans la gestion des « aspects sociaux » du nucléaire », in Wagener, Martin ; Francq, Bernard ; Scieur, Philippe ; Lits, Grégoire ; Vanneste, Damien (dir.) $\hat{E}$ tre curieux en sociologie. Presses universitaires de Louvain. p. 99-124.

Merzeau, Louise (2013), « L'intelligence des traces », Intellectica, n 59, p. 115-135.

Molinatti, Grégoire \& Simonneau, Lionel (2015), "A Socioenvironmental Shale Gas Controversy: Scientists' Public Communications, Social Responsibility and Collective Versus Individual Positions », Science Communication, n 37 (2), p. 190-216.

Olivier de Sardan, Jean-Pierre (2008), La rigueur du qualitatif. Les contraintes empiriques de l'interprétation socio-anthropologique. Louvain-la-Neuve : Academia-Bruylant.

Pascual Espuny, Céline (2014), «La société civile, de l'alerte à la controverse médiatisée ", Communication E Organisation, $\mathrm{n}^{\circ} 45$, p. 115-126.

Patinaux, Lény (2017), Enfouir des déchets nucléaires dans un monde conflictuel. Une histoire de la démonstration de sûreté de projets de stockage géologique, en France (1982-2013), Thèse de doctorat en histoire, Paris : EHESS.

Pestre, Dominique (2001), «Études sociales des sciences, politique et retour sur soi éléments », Revue du MAUSS, n 17, p. 180-196.

Quet, Matthieu (2008), "L'innovation éditoriale des revues de critique des sciences », Médiamorphoses, Hors série n 4, p. 225-230.

Quet, Matthieu (2014), «Pour une approche communicationelle des enjeux scientifiques et techniques », Canadian Journal of Communication, n 39 (4), p. 651-662.

Roqueplo, Philippe (1997), Entre savoir et décision, l'expertise scientifique, Paris : Editions Quæ.

Simonnot, Brigitte (2018), "Conduire des recherches en régime numérique : vers un cadre conceptuel de réflexion éthique ", in Balicco, Laurence ; Chartron, Ghislaine ; Clavier, Viviane ; Pailliart, Isabelle (dirs.) L'éthique en contexte info-communicationnel numérique. Déontologie, régulation, algorithme, espace public. Bruxelles : De Boeck Supérieur/ADBS, p. 11-21.

Théry, Irène (2005), « Expertises de service, de consensus, d'engagement : essai de typologie de la mission d'expertise en sciences sociales », Droit et société, n 60 (2), p. 311-327.

Weber, Max (1963), Le savant et le politique. Paris : Union Générale d'Éditions. 


\section{ANNEXE : COMPOSITION DU CORPUS}

Guéritte, Michel (2018), CIGéo Papers. 5 février, [en ligne], https://www.villesurterre.eu/ index.php?option=com_content\&view=article\&id=634:cigeo-papers\&catid=124:news\&Itemid $=214$

Le Hir, Pierre (2018), "Bure : l'impossible preuve scientifique de la sûreté », Le Monde, 8 février, [en ligne], https://www.lemonde.fr/planete/article/2018/02/07/centre-d-enfouissement-de-bure-l-impossible-preuve-scientifique-de-la-surete 5252802 3244.html

L., F. (2018) «Cigéo : une thèse qui fait parler », Le Journal de la Haute-Marne, 9 février.

Gacon, Julie (2018), «Bure, Flamanville... Peut-on évaluer le risque nucléaire ?» Dimanche, et après ?, France Culture, 11 février, [en ligne], https://www.franceculture.fr/ emissions/dimanche-et-apres/dimanche-et-apres-du-dimanche-11-fevrier-2018

Société française d'énergie nucléaire (2018), "Cigéo Papers ", retour sur la méthodologie de l'Andra. 27 février, [en ligne], http://www.sfen.org/rgn/cigeo-papers-methodologie-andra

Andra (2018), Rapport d'activités scientifiques et techniques, [en ligne], https://www.andra.fr/ sites/default/files/2018-09/RA\%20ANDRA\%202017 EXE-BD.pdf

Anon. (2018), «À Bure : «Aujourd'hui, c'est l'économie qui évalue ce qui constitue un risque nucléaire acceptable » », Grozeille, 9 mai, [en ligne], https://grozeille.co/bureeconomie-risque-nucleaire/

Assemblée Nationale (2018), Compte rendu de réunion de la commission d'enquête sur la sûreté et la sécurité des installations nucléaires. Audition de M. Leny Patinaux, auteur d'une thèse sur Cigéo, [en ligne], http://www.assemblee-nationale.fr/15/cr-cenucl/17-18/c1718035.asp

L’ensemble des sites a été consulté le 13 mars 2019. 